\title{
RATIONAL FUNCTIONS CERTIFY COMBINATORIAL IDENTITIES
}

\author{
HERBERT S. WILF AND DORON ZEILBERGER
}

\section{INTRODUCTION}

This paper presents a general method for proving and discovering combinatorial identities: to prove an identity one can present a certificate that consists of a pair of functions of two integer variables. To prove the identity, take the two functions that are given, check that condition (1) below is satisfied (a simple mechanical task), and check the equally simple fact that the boundary conditions (F1), (G1), (G2) below are satisfied. The identity is then proved.

Alternatively, one can present the identity itself, and a single rational function. To prove the identity the reader would then construct the pair of functions referred to above, and proceed as before (see $\$ 3$ below).

In this paper we present several one-line proofs of hypergeometric identities. All of these one-line proofs were found by using the method presented below, on computers that have strong symbolic manipulation packages. Once the proofs have been found, they can be checked by hand or on small personal computers that would need only minimal symbolic manipulation capability.

Not too long ago the world of combinatorial identities consisted of hundreds of individually proved relations (for a valuable collection of these see [10]), mostly involving binomial coefficients. As a result of ideas of $\mathrm{H}$. Bateman (see the introduction to [10]), G. Andrews [1], and others, it is widely recognized that most of these are special cases of relatively few hypergeometric identities, and attention is now being turned to methods of systematizing these higher level relationships.

Gosper [9] has shown how to find indefinite hypergeometric sums, where they exist, by quite a general procedure (see [11]). In this paper we describe a general attack on definite hypergeometric, and other, sums, continuing the program started in [13-15].

- The method can prove, in a unified way, virtually all known hypergeometric sum identities (and therefore legions of binomial coefficient identities too). It does this by means of certificates of proof, each of which consists of a pair of functions $(F, G)$ (a 'WZ-pair') that satisfy certain conditions, described below. As a by-product, each such pair

Received by the editors January 25, 1989.

1980 Mathematics Subject Classification (1985 Revision). Primary 05A19, 05A10.

The first author was supported in part by the U. S. Office of Naval Research.

The second author was supported in part by the U. S. National Science Foundation. 
$(F, G)$ will actually certify the truth of two identities, with no extra effort.

- The method can discover, in a unified way, new identities. We will show how, to any WZ-pair $(F, G)$, one can associate a dual pair $\left(F^{\prime}, G^{\prime}\right)$, that may produce one or two additional identities, which we will call the duals of the original ones. So altogether we will have not only the proof of the original identity, but the discovery and proof of two or three additional identities.

- But even more is true. Most hypergeometric identities involve a few auxiliary free parameters. By specializing these parameters, we get a countless number of new identities, that are however trivially implied by the original, more general identity. It turns out that the dual of a specialization is not, in general, a specialization of the dual. So the trivial process of specialization, composed with the new operation of dualization leads to the discovery of countless new identities, that a priori seem highly nontrivial.

For example many of Gosper's 'strange' identities, that were tackled one at a time in [7], turn out to be duals of specializations of classical identities (mostly those of Saalschütz and Dougall). Those identities in [7] whose duals are not specializations of classical identities are very possibly the result of iterating specialization and dualization several times. Dr. Stanton points out that [8] also identifies the 'strange' identities as duals of specializations of classical identities, in that case relative to a notion of 'duality' that is based on Lagrange inversion.

Another striking example of the insight gained by this new concept of the dual identity is the realization that Dixon's classical well-poised ${ }_{3} F_{2}$ (see, e.g., [2]) that has three free parameters is nothing but the dual of a certain specialization of the famous (four-parameter), balanced, Saalschütz identity. (Precisely, the dual of Dixon's

$$
{ }_{3} F_{2}\left[\begin{array}{ccc}
a & b & -n \\
& 1+a-b & 1+a+n
\end{array}\right]
$$

is the specialization

$$
{ }_{3} F_{2}\left[\begin{array}{ccc}
-a+n+1 & -a / 2+b & -n \\
& -a / 2+1 & b-a+1
\end{array}\right]
$$

of Saalschütz.)

\section{THE MAIN THEOREM}

The idea is this. Suppose we have two functions $F(n, k), G(n, k)$, defined for integer $k$ and integer $n \geq 0$, and suppose the following condition is satisfied:

$$
F(n+1, k)-F(n, k)=G(n, k+1)-G(n, k) \quad(\text { integers } n \geq 0, k) .
$$

We will then call $(F, G)$ a WZ-pair.

\footnotetext{
${ }^{1}$ Named after two complex variables.
} 
We show that under certain additional boundary conditions ((F1), (G1), (G2) below) we obtain a simple evaluation of the sum

$$
\sum_{k} F(n, k) \quad(n=0,1,2, \ldots) .
$$

We also obtain a simple evaluation of the associated sum

$$
\sum_{n \geq 0} G(n, k) \text {. }
$$

Thus we will obtain two identities, one for each member of the WZ-pair. The proofs of the identities will consist in simply verifying that the condition (1) is satisfied, along with the following boundary conditions:

(F1) For each integer $k$, the limit

$$
f_{k}=\lim _{n \rightarrow \infty} F(n, k)
$$

exists and is finite.

(G1) For each integer $n \geq 0, \lim _{k \rightarrow \pm \infty} G(n, k)=0$.

(G2) We have $\lim _{L \rightarrow \infty} \sum_{n \geq 0} G(n,-L)=0$.

Theorem A. Let $(F, G)$ satisfy (1). If $(\mathrm{G} 1)$ holds then we have the identity

$$
\sum_{k} F(n, k)=\text { const. } \quad(n=0,1,2, \ldots),
$$

(where 'const.' is found by putting $n=0)$. Further, if (F1), (G2) hold then we have the identity

$$
\sum_{n \geq 0} G(n, k)=\sum_{j \leq k-1}\left(f_{j}-F(0, j)\right),
$$

where $f$ is defined by (4).

Proof. Sum both sides of equation (1) from $k=-L$ to $k=K$. This gives

$$
\begin{aligned}
\Delta_{n}\left\{\sum_{k=-L}^{K} F(n, k)\right\} & =\sum_{k=-L}^{K}\left\{\Delta_{k} G(n, k)\right\} \\
& =G(n, K+1)-G(n,-L),
\end{aligned}
$$

where $\Delta_{n}$ and $\Delta_{k}$ are the forward difference operators that act on $n$ and $k$, respectively. If we let $K, L \rightarrow \infty$ and use (G1), we discover that $\sum_{k} F(n, k)$ is independent of $n \geq 0$, and (5) is proved.

Similarly if we sum both sides of (1) from $n=0$ to $N$, we obtain

$$
F(N+1, k)-F(0, k)=\Delta_{k}\left\{\sum_{n=0}^{N} G(n, k)\right\} \text {. }
$$

Taking the limit as $N \rightarrow \infty$ and using (F1) yields

$$
f_{k}-F(0, k)=\Delta_{k}\left\{\sum_{n \geq 0} G(n, k)\right\} \text {. }
$$


Finally, if we sum from $-L$ to $k-1$, let $L \rightarrow \infty$, and use (G2), we get (6).

We remark that trivially all WZ-pairs can be constructed as follows. First choose any function $\Phi(n, k)$ (the 'potential function'). Then set $F=\Delta_{k} \Phi$ and $G=\Delta_{n} \Phi$. This enables us to manufacture identities all day long, and if we want hypergeometric identities, all we have to do is choose $\Phi$ to be of closed form (see below). However, finding interesting identities that way is not so easy.

\section{EXAMPLES OF CERTIFICATION}

Example 1. To begin with a simple case, consider the WZ-pair

$$
\begin{aligned}
& F(n, k)=\left(\begin{array}{l}
n \\
k
\end{array}\right) / 2^{n}, \\
& G(n, k)=-\left(\begin{array}{c}
n \\
k-1
\end{array}\right) / 2^{n+1} .
\end{aligned}
$$

A quick check of (1), (F1), (G1), (G2) shows that they are all satisfied, and that $f_{k}=0$ for all $k$. Then (5) and (6) become the two identities

$$
\sum_{k}\left(\begin{array}{l}
n \\
k
\end{array}\right) / 2^{n}=1 \quad(n \geq 0)
$$

and

$$
\sum_{n \geq 0}\left(\begin{array}{c}
n \\
k-1
\end{array}\right) / 2^{n+1}=1 \quad(k \geq 1) .
$$

Example 2. Consider the pair

$$
\begin{aligned}
& F(n, k)=\frac{n !^{2}}{k !^{2}(a-k) !(n-k) !(n+a) !}, \\
& G(n, k)=-\frac{a-k+1}{n+a+1} F(n, k-1) .
\end{aligned}
$$

As soon as we check that the conditions (1), (F1), (G1), (G2) are satisfied, again with all $f_{k}=0$, we have a proof of Vandermonde's identity (the sum is finite)

$$
\sum_{k}\left(\begin{array}{l}
a \\
k
\end{array}\right)\left(\begin{array}{l}
n \\
k
\end{array}\right)=\left(\begin{array}{c}
n+a \\
a
\end{array}\right)
$$

as well as of the identity (the sum is infinite)

$$
\sum_{n} \frac{\left(\begin{array}{c}
n \\
k
\end{array}\right)}{\left(\begin{array}{c}
n+a+1 \\
n
\end{array}\right)}=\frac{a+1}{(k+1)\left(\begin{array}{c}
a \\
k+1
\end{array}\right)} \quad(\text { integer } a>k \geq 0) .
$$

Example 3. The functions

$$
\begin{aligned}
& F(n, k)=\left(\begin{array}{c}
2 n \\
n
\end{array}\right)^{-1}\left(\begin{array}{l}
n \\
k
\end{array}\right)^{2}, \\
& G(n, k)=-\frac{(3 n-2 k+3)}{2(2 n+1)} F(n, k-1)
\end{aligned}
$$


are a WZ-pair, and once more all $f_{k}=0$. Thus we have the two identities

$$
\begin{gathered}
\sum_{k}\left(\begin{array}{l}
n \\
k
\end{array}\right)^{2}=\left(\begin{array}{c}
2 n \\
n
\end{array}\right) \quad(n \geq 0), \\
\sum_{n \geq 0} \frac{(3 n+1-2 k)}{(2 n+1)\left(\begin{array}{c}
2 n \\
n
\end{array}\right)}\left(\begin{array}{l}
n \\
k
\end{array}\right)^{2}=2 \quad(k \geq 0) .
\end{gathered}
$$

Example 4. To prove the identity of Saalschütz we need only check that the two functions

$$
\begin{aligned}
& F(n, k)=\frac{(a+k-1) !(b+k-1) ! n !(-a-b+c+n-1-k) !(c+n-1) !}{k !(n-k) !(c+k-1) !(c-a+n-1) !(c-b+n-1) !} \\
& G(n, k)=-\frac{(b+k-1)(a+k-1)}{(c-b+n)(c-a+n)} F(n, k-1)
\end{aligned}
$$

are a WZ-pair and satisfy $(F 1),(G 1),(G 2)$. In this case

$$
f_{k}=\lim _{n \rightarrow \infty} F(n, k)=\frac{(a+k-1) !(b+k-1) !}{k !(c+k-1) !} .
$$

Hence we have a proof not only of Saalschütz's identity (which is (5) in this case)

$$
{ }_{3} F_{2}\left[\begin{array}{ccc}
a, & b, & -n \\
& c, & 1+a+b-c-n
\end{array}\right]=\frac{(c-a)_{n}(c-b)_{n}}{(c)_{n}(c-a-b)_{n}}
$$

but also of the identity (6), which takes the form

$$
\begin{aligned}
{ }_{3} F_{2}\left[\begin{array}{rrr}
k & c-a-b & c+k-1 \\
& c-b+k & c-a+k
\end{array}\right] \\
\quad=\frac{(c-b)_{k}(c-a)_{k}}{(a)_{k}(b)_{k}}\left\{1-\frac{(c-a-b)_{a}}{(c-a)_{a}} \sum_{j=0}^{k-1} \frac{(a)_{j}(b)_{j}}{j !(c)_{j}}\right\},
\end{aligned}
$$

in which the sum on the left is infinite. This identity is essentially in $\S 10.4$ of Bailey [2]. We will find, generally, that the companion identity (6) of a hypergeometric identity is of the type that relates a hypergeometric function to a partial sum of the series for another hypergeometric function. Many such identities are known, and our method shows a natural reason for their existence.

Example 5. A complete proof of the identity of Clausen [11, p. 525]

$$
{ }_{4} F_{3}\left[\begin{array}{cccc}
a, & b, & \frac{1}{2}-a-b-n, & -n \\
& \frac{1}{2}+a+b, & 1-a-n, & 1-b-n
\end{array}\right]=\frac{(2 a)_{n}(a+b)_{n}(2 b)_{n}}{(2 a+2 b)_{n}(a)_{n}(b)_{n}}
$$

results from checking the following WZ-pair:

$$
\begin{aligned}
& F(n, k)=\phi(k) \phi(n-k) \psi(n), \\
& G(n, k)=-\frac{(b+k-1)(a+k-1)(2 b+2 a+3 n-2 k+2)}{(2 b+n)(a+b+n)(2 a+n)} F(n, k-1),
\end{aligned}
$$


where

$$
\begin{aligned}
\phi(t) & =\frac{(a+t-1) !(b+t-1) !}{t !\left(-\frac{1}{2}+a+b+t\right) !} \\
\psi(t) & =\frac{\left(t+a+b-\frac{1}{2}\right) ! t !(t+2 a+2 b-1) !}{(t+2 a-1) !(t+a+b-1) !(t+2 b-1) !} .
\end{aligned}
$$

The second identity that the same pair proves is

$$
\sum_{n \geq 0} \frac{(2 b+2 a+3 n-2 k) \phi(n-k) \psi(n)}{(n+2 b)(n+2 a)(n+a+b)}=\frac{\phi(0)^{2} \psi(0)-\sum_{j=0}^{k} \phi(j)}{(k+a)(k+b) \phi(k)} \quad(k \geq 0),
$$

where $\phi$ and $\psi$ are as shown above.

Example 6. The identity of Dixon is

$$
\sum_{k}(-1)^{k}\left(\begin{array}{l}
n+b \\
n+k
\end{array}\right)\left(\begin{array}{l}
n+c \\
c+k
\end{array}\right)\left(\begin{array}{l}
b+c \\
b+k
\end{array}\right)=\frac{(n+b+c) !}{n ! b ! c !}
$$

The WZ-pair that works here is

$$
\begin{aligned}
& F(n, k)=\frac{(-1)^{k}(n+b) !(n+c) ! n !}{(n-k) !(n+k) !(b-k) !(b+k) !(c-k) !(c+k) !(n+b+c) !}, \\
& G(n, k)=\frac{(c+1-k)(b+1-k)}{2(n+k)(n+b+c+1)} F(n, k-1) .
\end{aligned}
$$

Here we find that the $f_{k}$ of (4) are

$$
f_{k}=\frac{(-1)^{k}}{(b-k) !(b+k) !(c-k) !(c+k) !} .
$$

Then the general identity (5) of the theorem becomes Dixon's identity, above. We leave the identity that comes from (6), in this case, to the reader.

Remark. The certification can be done in another way. Instead of giving the WZ-pair $(F, G)$, one can state the identity to be proved in its familiar form, say as

$$
\sum_{k} U(n, k)=r h s(n) \quad(n \geq 0 ; \operatorname{rhs}(n) \neq 0) .
$$

To certify it, one need only give the rational function $R(n, k)$ that satisfies $G(n, k)=R(n, k) F(n, k-1)$; nothing else.

The reader who wished to check the certificate would proceed as follows:

(a) Divide the identity through by $r h s(n)$, obtaining the first member of the WZ-pair as $F(n, k)=U(n, k) / r h s(n)$.

(b) Find $G(n, k)=R(n, k) F(n, k-1)$.

(c) Check that conditions (1), (F1), (G1), (G2) are satisfied.

(d) Verify that the identity is true when $n=0$.

Hence one can imagine a book full of identities and their proofs, where each proof consists of just giving a certain rational function. In [12] there are 54 examples of such certifications of binomial coefficient identities. Here are some hypergeometric examples. 
Theorem. Dixon's identity above is true.

Proof. Take $R(n, k)=(c+1-k)(b+1-k) /(2(n+k)(n+b+c+1))$.

Theorem. The 'strange' identity (1.4) of Gessel-Stanton [7]

$$
\sum_{k}\left(\frac{3}{4}\right)^{k} \frac{\left(k+3 a-\frac{1}{2}\right) !\left(k-3 a-\frac{1}{2}\right) !(3 n-k) !}{k !(n-k) !\left(k-\frac{1}{2}\right) !}=\frac{(3 n) !\left(n-a-\frac{1}{2}\right) !\left(n+a-\frac{1}{2}\right) !}{n !\left(n-\frac{2}{3}\right) !\left(n-\frac{1}{3}\right) !}
$$

is true.

Proof. Take

$$
R(n, k)=\frac{1}{9} \frac{(6 a+2 k-1)(6 a-2 k+1)}{(2 n+2 a+1)(2 n-2 a+1)} .
$$

We remark that here the companion identity (6) is new to us. It is

$$
\begin{aligned}
\sum_{n \geq 0} \frac{n !(3 n-k) !\left(n-\frac{2}{3}\right) !\left(n-\frac{1}{3}\right) !}{(n-k) !(3 n) !\left(n-a+\frac{1}{2}\right) !\left(n+a+\frac{1}{2}\right) !} \\
=\frac{9 k !\left(k-\frac{1}{2}\right) !}{\left(\frac{3}{4}\right)^{k}\left(k+3 a+\frac{1}{2}\right) !\left(k-3 a+\frac{1}{2}\right) !} \\
\quad \times\left\{2 \sqrt{\frac{\pi}{3}} \frac{\cos (\pi a)}{\cos (3 \pi a)}-\sum_{j \leq k} \frac{\left(\frac{3}{4}\right)^{j}\left(j+3 a-\frac{1}{2}\right) !\left(j-3 a-\frac{1}{2}\right) !}{j !\left(j-\frac{1}{2}\right) !}\right\} .
\end{aligned}
$$

Theorem. The 'strange' identity (1.7) of [7], which asserts that

$$
\begin{gathered}
\sum_{k} \frac{\left(k+\frac{2}{3} a\right)\left(n+k+a+d-\frac{1}{2}\right) !(k+a-d-1) !(2 n+2 d-k) !(k+2 a-1) !(k+2 b-1) !(k-2 b) !}{\left(k+a+b-\frac{1}{2}\right) !(k+2 d) !(2 n+k+2 a) ! k !(n-k) !(k+a-b) !} \\
=\frac{\left(n+a+d-\frac{1}{2}\right) !\left(n+b+d-\frac{1}{2}\right) !(n+d-b) !}{n !\left(n+a+b-\frac{1}{2}\right) !(n+a-b) !}
\end{gathered}
$$

is true.

Proof. Take $R(n, k)$ to be the rational function

$$
-\frac{(4 n+2 d+2 a+3)(k+2 b-1)(k-2 b)(k+2 a-1)(k+a-d-1)(2 n+2 k+2 a+2 d-1)}{(2 n+k+2 a)(2 n+2 d+2 a+1)(3 k+2 a-3)(n+d-b+1)(2 n+2 b+2 d+1)(2 n+k+2 a+1)} \cdot
$$

Theorem. The 'strange' identity (1.2) of [7]

$$
\begin{gathered}
\sum_{k} \frac{\left(-\frac{1}{4}\right)^{k}(k+2 a-1) !(k+2 b-1) !(k-2 b) !\left(k+\frac{2}{3} a\right)}{k !(n-k) !(k+a-b) !\left(k+a+b-\frac{1}{2}\right) !(2 n+k+2 a) !} \\
=\frac{\left(n+a-\frac{1}{2}\right) !(n+a) !}{n !(2 n+2 a) !\left(n+a+b-\frac{1}{2}\right) !(n+a-b) !}
\end{gathered}
$$

is true.

Proof. Take

$$
R(n, k)=-\frac{(k+2 b-1)(k-2 b)(k+2 a-1)}{4(2 n+k+2 a+1)(2 n+k+2 a)(3 k+2 a-3)} .
$$


Theorem. The ${ }_{7} F_{6}$ identity of Dougall

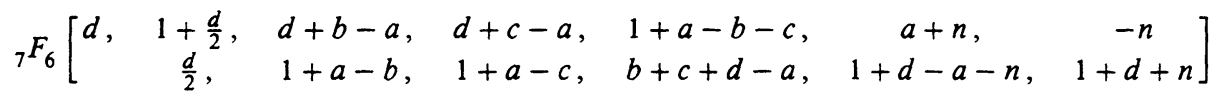

$$
\begin{aligned}
& =\frac{(d+1)_{n}(b)_{n}(c)_{n}(1+2 a-b-c-d)_{n}}{(a-d)_{n}(1+a-b)_{n}(1+a-c)_{n}(b+c+d-a)_{n}}
\end{aligned}
$$

is true.

Proof. Just take $R(n, k)$ to be

$-\frac{(k-b-c+a)(k+d-1)(k+b+d-a-1)(k+c+d-a-1)(n+k+a-1)(2 n+a+1)}{(2 k+d-2)(n+a)(n+b)(n+c)(n-b-c-d+2 a-1)(k+n+d)}$.

\section{SHadows}

In the following we maintain the convention that the range of $n$ is the set of nonnegative integers, while the range of $k$ is the set of all integers.

Definition. A discrete function $f(n)$ is said to be of closed form if $f(n+1) / f(n)$ is a rational function of $n$.

It is easily seen that any closed form function $f$ has the form

$$
x^{n} \frac{A(n)}{B(n)} \frac{\prod_{i}\left(a_{i} n+b_{i}\right) !}{\prod_{i}\left(a_{i}^{\prime} n+b_{i}^{\prime}\right) !} .
$$

Definition. A function $F(n, k)$ is said to be of closed form (CF) if both $R(n, k)=F(n+1, k) / F(n, k)$ and $R^{\prime}(n, k)=F(n, k+1) / F(n, k)$ are rational functions, of $n$ and of $k$, respectively.

Note that $R(n, k)$ and $R^{\prime}(n, k)$ plus some initial conditions determine $F(n, k)$ uniquely. However $R$ and $R^{\prime}$ must satisfy the obvious compatibility condition

$$
R(n, k+1) R^{\prime}(n, k)=R(n, k) R^{\prime}(n+1, k) .
$$

Definition. Two discrete functions $F(n, k)$ and $F^{\prime}(n, k)$, defined in different regions of the lattice, are equivalent if they produce the same $\left(R, R^{\prime}\right)$.

Example. In one variable, $n$ ! (for $n \geq 0$ ) is equivalent to $(-1)^{n} /(-n-1)$ ! (defined for $n<0)$. In two dimensions $(a n+b k+c)$ !, where $a$ and $b$ are integers, is equivalent to

$$
\frac{(-1)^{a n+b k}}{(-a n-b k-c-1) !}
$$

Also, $\left(\begin{array}{l}n \\ k\end{array}\right)$ is equivalent to

$$
(-1)^{n+k}\left(\begin{array}{l}
-k-1 \\
-n-1
\end{array}\right)
$$

Definition. The shadow $\widetilde{F}$ of a CF $F(n, k)$ of the form

$$
x^{n} y^{k} \text { (Rational Function) } \times \frac{\prod\left(a_{i} n+b_{i} k+c_{i}\right) !}{\prod\left(a_{i}^{\prime} n+b_{i}^{\prime} k+c_{i}^{\prime}\right) !}
$$


is obtained by replacing every factor $(a n+b k+c)$ ! by

$$
(-1)^{a n+b k} /(-a n-b k-c-1) !
$$

if $a+b \neq 0$, and leaving it alone if $a+b=0$.

For example, the shadow of $\left(\begin{array}{l}n \\ k\end{array}\right)$ is $(-1)^{n+k}\left(\begin{array}{l}-k-1 \\ -n-1\end{array}\right)$.

Theorem B. Let $(F, G)$ be a WZ-pair, and suppose that $G(n, k) / F(n, k-1)$ is a rational function of $n$ and $k$. Then

$$
\left(F^{\prime}(n, k), G^{\prime}(n, k)\right):=(\widetilde{G}(-k-1,-n), \widetilde{F}(-k,-n-1))
$$

is also a WZ-pair.

Proof. The pair $(\widetilde{F}, \widetilde{G})$ is equivalent to the original pair $(F, G)$, and therefore it satisfies equation (1), though not for $n \geq 0$, but instead for $n<0$. The flips of variables and functions shown in (7) transform the domain of $n$ back to the nonnegative integers, while preserving the fact that $(1)$ is satisfied.

The map $(F, G) \rightarrow\left(F^{\prime}, G^{\prime}\right)$ is an involution, hence we call $\left(F^{\prime}, G^{\prime}\right)$ the dual of the WZ-pair $(F, G)$.

While the dual pair certainly satisfies the condition (1), it need not satisfy all of (F1), (G1), (G2). Depending on those outcomes, we may obtain only one, or both, of the identities (5), (6) as dual identities. The examples in the next section illustrate this.

\section{EXAMPLES OF DUAL IDENTITIES}

Example 1'. With the $F$ and $G$ of Example 1 above, the dual WZ-pair is

$$
\left(F^{\prime}(n, k), G^{\prime}(n, k)\right)=(-1)^{n+k} 2^{k}\left(\left(\begin{array}{l}
n \\
k
\end{array}\right),\left(\begin{array}{c}
n \\
k-1
\end{array}\right)\right) .
$$

Condition (F1) is not satisfied, but (G1), (G2) are, so by Theorem A we have identity (5), which is the statement $\sum_{k} F^{\prime}(n, k)=$ const.

Example $3^{\prime}$. If we use the $(F, G)$ of Example $3, \S 3$ above, then we find that the dual pair is

$$
\begin{aligned}
& F^{\prime}(n, k)=\frac{3 k-2 n}{2}\left(\begin{array}{l}
n \\
k
\end{array}\right)^{2}\left(\begin{array}{c}
2 k \\
k
\end{array}\right), \\
& G^{\prime}(n, k)=\frac{k}{2}\left(\begin{array}{c}
n \\
k-1
\end{array}\right)^{2}\left(\begin{array}{c}
2 k \\
k
\end{array}\right) .
\end{aligned}
$$

The boundary conditions (G1), (G2) are satisfied, and so we have that $\sum_{k} F^{\prime}(n, k)$ is independent of $n \geq 0$. Since it is 0 when $n=0$, we have proved the dual identity

$$
\sum_{k}(3 k-2 n)\left(\begin{array}{l}
n \\
k
\end{array}\right)^{2}\left(\begin{array}{c}
2 k \\
k
\end{array}\right)=0 \quad(n=0,1, \ldots) .
$$

Example $4^{\prime}$. In the case of the Saalschütz WZ-pair, of Example 4, an easy calculation shows that the dual $F$ is

$$
F^{\prime}(n, k)=-\frac{(n-k+c-a-b-1) !(k-c+b) !(k-c+a) !(n !)(n+1-c) !}{(n-b) !(n-a) !(k) !(k-c+1) !(n-k) !} .
$$


We observe that this $F^{\prime}$ is, aside from a sign and a renaming of the parameters $a, b, c$, the same as the original $F$. Hence the identity of Saalschütz is self dual.

Example 6'. The dual of Dixon's identity (Example 6, $\S 3$ above) is quite interesting. We find that

$$
F^{\prime}(n, k)=\frac{(-1)^{k}(n+k) !(k-b-c-1) !(-n-b-1) !(n-b) !(-n-c-1) !(n-c) !}{2(k-b) !(k-c) ! k !(n-k) !} .
$$

Also

$$
G^{\prime}(n, k)=\frac{(-1)^{k}(n+k) !(-n-b-2) !(n-b) !(-n-c-2) !(n-c) !(k-b-c-1) !}{(k-b-1) !(k-c-1) !(k-1) !(n-k-1) !} .
$$

Now (F1), (G1), and (G2) all hold. The identity (5) for $F^{\prime}$ can be written in the following form, after renaming the free variables $b, c$ to $-b,-c$ :

$$
\sum_{k} \frac{(-1)^{k}\left(\begin{array}{c}
n \\
k
\end{array}\right)\left(\begin{array}{c}
k+b+c-1 \\
k+c
\end{array}\right)}{\left(\begin{array}{c}
b+k \\
n+k
\end{array}\right)}=\frac{2(b-n)}{b+c} \frac{\left(\begin{array}{c}
b+c \\
b
\end{array}\right)\left(\begin{array}{c}
2 c-1 \\
n+c
\end{array}\right)}{\left(\begin{array}{c}
2 c \\
c
\end{array}\right)\left(\begin{array}{c}
n+b \\
b
\end{array}\right)}
$$

where $0 \leq n \leq b$, and it is a specialization of Saalschütz's.

Example $7^{\prime}$. The first identity of the pair that is dual to the identity (1.4) of [7] is the specialization

$$
{ }_{3} F_{2}\left[\begin{array}{ccc}
\frac{1-2 n}{3}, & -\frac{2 n}{3}, & -\frac{2 n+1}{3} \\
& -n+a+\frac{1}{2}, & -n-a-\frac{1}{2}
\end{array}\right]
$$

of Saalschütz's identity.

Example $8^{\prime}$. The first identity of the pair that is dual to the identity (1.7) of [7] is the specialization

$$
F\left[\begin{array}{ccccccc}
\frac{1}{2}-a-d & \frac{5}{4}-\frac{a+d}{2} & b-d & \frac{n+1}{2}-a & \frac{n}{2}-a+1 & \frac{1}{2}-b-d & -n \\
& \frac{1}{4}-\frac{a+d}{2} & \frac{3}{2}-a-b & 1-d-\frac{n}{2} & \frac{1-n}{2}-d & b-a+1 & n-a-d+\frac{3}{2}
\end{array}\right]
$$

of Dougall's ${ }_{7} F_{6}$ identity.

Example 9'. The first identity of the pair that is dual to the identity (1.2) of [7] is the specialization

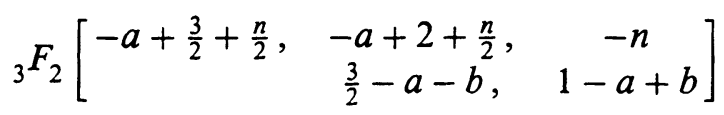

of Saalschütz's identity.

In each of the last three examples it should be noted that since duality is symmetric, the three identities of [7] are proved above by the process of specialization of known identities followed by dualization. They were proved in $\S 3$ directly by the certification process.

\section{THE SOUL IN THE MACHINE}

Every identity of the form $\sum_{k} F(n, k)=r h s(n)$, where $F(n, k)$ and $r h s(n)$ are of $\mathrm{CF}$ and $r h s(n) \neq 0$ is equivalent to one in which $r h s(n)=1$ : simply divide through by $r h s(n)$. Does every such $F$ have a mate $G$ ? 
Not always, but very often, and there is something weaker that is always guaranteed. In [14] it was shown, using the general theory of [13], that for every holonomic $F(n, k)$, there exists a CF $G(n, k)$, that is moreover of the form $R(n, k) F(n, k)$ for some rational function $R(n, k)$, such that for some $L$ and polynomials (in $n) a_{0}(n), a_{1}(n), \ldots, a_{L}(n)$ we have

$$
\begin{aligned}
a_{0}(n) & F(n, k)+a_{1}(n) F(n+1, k)+\cdots+a_{L}(n) F(n+L, k) \\
& =G(n, k+1)-G(n, k) .
\end{aligned}
$$

It follows upon summing w.r.t. $k$ that $S(n)=\sum_{k} F(n, k)$ satisfies the recurrence

$$
a_{0}(n) S(n)+a_{1}(n) S(n+1)+a_{2}(n) S(n+2)+\cdots+a_{L}(n) S(n+L)=0,
$$

where the $a_{i}$ are polynomials in $n$. To prove that $S(n)$ is indeed 1 (or a constant) all we have to check is that 1 is also a solution of the recurrence, i.e. that $\sum_{i} a_{i}(n)=0$, and that $S(n)=1$ for $n=0,1, \ldots, L-1$ (assuming that $a_{L}(n)$ has no positive integral roots).

So for holonomic summands one always has the certificate

$$
\left(G(n, k), a_{0}(n), \ldots, a_{L}(n)\right)
$$

and all we have to do is to verify that (8) is satisfied. If we are lucky the recurrence is first order (i.e. $L=1$ ), and $a_{0}+a_{1}=0$, so, by dividing through by $a_{0}$ we get (1). The amazing thing is that it happens so often. We know of only two cases where $L>1$.

So thanks to the general theory of [13], that is based on I. N. Bernstein's theory of holonomic systems (see [3]), we know that $G(n, k)$ of CF exists, as do the the $a_{i}$ 's. Hoping that indeed $L=1$, we have to find $G(n, k)$ of CF that satisfies (1), where $F(n, k)$ is given. This is done by Gosper's beautiful algorithm [9], that decides for us when such a $G$ exists, and when it does, finds it.

It is amazing that we are lucky so often, and whenever we are, we get in addition to a proof of the original identity, some brand new identities, complete with proofs.

\section{ACKNOWLEDGMENTS}

We would like to thank Shalosh B. Ekhad for diligently producing the WZpairs in this paper (and scores of others) by running a MAPLE progam that was based on the algorithm described in [14], that uses Gosper's algorithm [9] as a subroutine.

We would like to thank Drs. Richard Askey and Donald Knuth for stimulating discussions that have been most helpful in the preparation of this paper.

\section{REFERENCES}

1. George E. Andrews, Applications of basic hypergeometric functions, SIAM Rev. 16 (1974), 441-484.

2. W. N. Bailey, Generalized hypergeometric series, Cambridge Tracts in Mathematics, Vol. 32, Cambridge Univ. Press, London, 1935. (Reprinted by Hafner, New York, 1964.)

3. J. E. Björk, Rings of differential operators, North-Holland, Amsterdam, 1979. 
4. Shalosh B. Ekhad, A very short proof of Dixon's theorem, J. Combin. Theory Ser. A (to appear).

5. Math. Soc. 107 (1989), 1143.

6. Shalosh B. Ekhad and Doron Zeilberger, $A$ 21st century proof of Dougall's hypergeometric sum identity, J. Math. Anal. Appl. (to appear).

7. I. Gessel and D. Stanton, Strange evaluations of hypergeometric series, SIAM J. Math. Anal. 13 (1982), 295-308.

8. _ Applications of q-Lagrange inversion to basic hypergeometric series, Trans. Amer. Math. Soc. 277 (1983), 173-201.

9. R. William Gosper, Jr., Decision procedure for indefinite hypergeometric summation, Proc. Nat. Acad. Sci. U.S.A. 75 (1978), 40-42.

10. Henry W. Gould, Combinatorial identities, Morgantown, West Virginia, 1972.

11. R. L. Graham, D. E. Knuth, and O. Patashnik, Concrete mathematics, Addison-Wesley, Reading, MA, 1989.

12. Herbert S. Wilf, 54 computer-generated proofs of binomial coefficient identities (to appear).

13. Doron Zeilberger, $A$ holonomic systems approach to special function identities (to appear).

14. __ $A$ fast algorithm for proving terminating hypergeometric identities (to appear).

15. __ The method of creative telescoping (to appear).

Department of Mathematics, University of Pennsylvania, Philadelphia, PennsylvaNIA $19104-6395$

Department of Mathematics, Drexel University, Philadelphia, Pennsylvania 19104 